\title{
Seroprevalence of IgG Antibodies of Toxoplasma in Healthy Voluntary Blood Donors in a Tertiary Care Hospital
}

\author{
Sundararajan Thangavel $^{1}$, Gomathi Manian ${ }^{2} *$ and Neelaveni Duraisamy $^{3}$ \\ Department of Microbiology, Government Mohan Kumaramangalam, Medical College, \\ Salem, Tamil Nadu, India \\ *Corresponding author
}

\section{Ke ywords \\ Toxoplasma gondii, Seroprevalence, IgG antibody, ELISA \\ Article Info \\ Accepted: \\ 07 February 2019 \\ Available Online: \\ 10 March 2019}

\begin{abstract}
A B S T R A C T
Many parasitic diseases can be transmitted by blood transfusion. Among the protozoal diseases transmitted by blood transfusion in India, the most important is malaria followed by toxoplasmosis. Screening for malaria is mandatory in India. Seroprevalence rates in the general population in India vary from a low of $1 \%$ to a high of $57 \%$. Aim of the study is to estimate the prevalence of Toxoplasma gondii $\mathrm{IgG}$ antibodies in healthy voluntary blood donors in a tertiary care hospital in and around Salem. This Cross-sectional study was conducted in the Department of Microbiology over a period of 3 months (March-May 18). Healthy voluntary blood donors from blood donation camps conducted at different centres in and around Salem, TamilNadu. The samples which were collected from healthy donors screened for Toxoplasma specific IgG antibodies by ELISA using commercially available kits (Merilisa, Meril Diagnostics). In the total of 180 samples which were tested for Toxoplasma IgG antibodies, the total number of serum positive samples were $40(22.2 \%)$ by ELISA. which is more common in the age group 31-40yrs. 12 (27.3\%) followed by 21 30 yrs $14(23.3 \%))$. In the present study, most of the participants were living in Uran areas (75\%), blood group A\&O (30\%) followed by B (25\%) and most of them were nonvegetarians $(75 \%)$. Toxoplasma is one of the most important congenitally transmitted infection. To ensure availability of toxoplasma seronegative blood for transfusion, a national policy of screening of blood and blood products for toxoplasma may need to be included along with other mandatory screening tests.
\end{abstract}

\section{Introduction}

Many parasitic diseases can be transmitted by blood transfusion. Among the protozoal diseases transmitted by blood transfusion in India, the most important is malaria followed by toxoplasmosis. Screening for malaria is mandatory in India. Although toxoplasmosis occurs worldwide, seropositivity levels vary widely among different regions of the globe (from a low of $4.1 \%$ in Thailand ${ }^{(1)}$ to $75 \%$ in Brazil $\left.{ }^{(2)}\right)$. Seroprevalence rates in the general population in India vary from a low of $1 \%{ }^{(6)}$ to a high of $57 \%{ }^{(7)}$. Toxoplasma gondii completes its life cycle in 2hosts.Cats and other felines are the definitive host. Man and 
other mammals are the intermediate hosts in which T.gondiii has 2 types of life cycles:Enteric cycle and Exoenteric cycle. Toxoplasma gondii is an obligate intracellular protozoal parasite, causes toxoplasmosis. Toxoplasma gondii, which can multiply in any nucleated cell of human body. The infection can remain dormant for many years and by activation to cause clinical symptoms. Transmission of infection occurs by more than one route. Food borne transmission (consuming undercooked contaminated meat), animal to human transmission (ingesting oocyst shed in the faeces of infected cats) and vertical transmission from mother to fetus through the placenta during delivery. T.gondiii can also be transmitted via blood transfusion or organ transplantation from infected donors.

The danger of transmission of T.gondiii infection to recipients is more by transfusing blood from asymptomatic, apparently healthy donors. Screening for toxoplasma in blood and blood product is not mandatory in India. This could pose a great risk to the recipients of blood and blood products. Studying blood donor population is a valuable approach to determine epidemiological characteristics in adults of a community and might provide findings that could be found in the general adult populations of the same community. The overall seropositive of T.gondii antigen by IgG ELISA was $13.14 \%$ in and around Tirunelveli district in Tamil $\mathrm{Nadu}^{(15)}$. The toxoplasma seroprevalence rate of $20.3 \%$ was seen in healthy voluntary blood donors from Karnataka $^{(11)}$.This study is aimed to assess the seroprevalence of $T$.gondiii infection in the blood collected from healthy voluntary donors from Urban areas of Tamil Nadu.

To estimate the prevalence of Toxoplasma gondii IgG antibodies in healthy voluntary blood donors in a tertiary care hospital in and around Salem.

\section{Materials and Methods}

This Cross-sectional study was conducted in the Department of Microbiology over a period of 3 months (March-May 18). A total of 180 samples were collected in and around Salem. Healthy voluntary blood donors from blood donation camps conducted at different centres in and around Salem, Tamil Nadu. The donors are healthy adults, screened routinely by physical examination with no history of infections in the recent past. Inclusion criteria included were: The healthy adult donors (>18 yrs) without any history of recent infections. Serum samples for healthy blood donors were taken. Exclusion criteria include: The donor samples which were hepatitis and HIV serum positive were excluded from the study.

The donors were healthy adults screened routinely by physical examination with no history of infection in the recent past. The samples which were collected from healthy donors screened for Toxoplasma specific IgG antibodies by ELISA using commercially available kits (Merilisa, Meril Diagnostics).

MERIL TOXO IgG ELISA is based on indirect ELISA reactios. The wells were precoated with T.gondiii antigen, once the sample was added, anti-Toxo (IgG) if present, binds to pre-coated antigens.

After incubation and wash procedures, Enzyme conjugate was added and the antihuman IgG inside binds to anti-Toxo (IgG) attached to the solid phase in the previous step. After another incubation and wash procedures, add Substrate A and Substrate B to initiate a chromogenic reaction. Once the colour development was completed, the stop solution was added and then the absorbance of each sample was read. The colour intensity is directly proportional to anti-Toxo (IgG) concentration. 
Samples with cutoff value $>0.18$ were considered as positive and others as negative. The results were interpreted as positive or negative as per manufacturer's instruction. The demographic details noted were age, sex, domicile, dietary habits and blood group. The discrete variables were expressed as percentage.

\section{Results and Discussion}

In the total of 180 samples which were tested for Toxoplasma $\mathrm{IgG}$ antibodies, the total number of serum positive samples were 40 (22.2\%) by ELISA. which is more common in the age group 31-40yrs. 12 (27.3\%) followed by $21-30$ yrs 14 (23.3\%), up to 20 yrs $12(20 \%)$ and 41-50yrs 2(16.7\%).

In the present study, a total of 180 blood donors were included, out of which 40 were found to be positive for toxoplasma $\operatorname{IgG}$ antibodies. Most of the participants were, aged $31-40 y r s(27.3 \%)$, living in Uran areas (75\%), blood group A\&O (30\%) followed by B $(25 \%)$ and most of them were non-vegetarians
(75\%). In our study, the toxoplasma seroprevalence rate in healthy voluntary donors from the Salem district was $22.2 \%$. Sreoprevalence rates in the general population in India vary from a low of $1 \%{ }^{(6)}$ to a high of $57 \%{ }^{(7)}$. Study done at Thirunelveli district was $13.4 \% .{ }^{(15)}$ Sundar et al., from Karnataka reported the seroprevalence rate of $\operatorname{IgG} 20.3 \%{ }^{(11)}$ which is similar to the present study. In this present study, the seroprevalence rate in urban area was $75 \%$ and in rural $25 \%$. In contrast, the seroprevalence rate reported from urban and rural areas from Chandigarh was $4.7 \%^{(8)}$ and hospital based study from Jodhpur in Rajasthan was $17.2 \%{ }^{(9)}$ which was low when compared to the present study. ${ }^{(8)}$ Meisheri et al., from Mumbai reported much higher seroprevalence $(30.9 \%)$ in general population. ${ }^{(10)}$ These variations could be related to socio-cultural habits, geographic and environmental factors, the state of general hygiene in the society and the routes of transmission. The risk factors associated with anti T.gondiii antibodies were age, gender, residence, dietary habits and blood group (Table 1-3). ${ }^{(15)}$

Table.1 Overall percentage of Toxoplasma IgG antibodies $(n=180)$

\begin{tabular}{|l|l|l|}
\hline \multicolumn{2}{|c|}{ Number } & $\begin{array}{l}\text { Percentag } \\
\text { e }\end{array}$ \\
\hline Positive & 40 & 22.2 \\
\hline Negative & 140 & 77.8 \\
\hline Total & 180 & 100 \\
\hline
\end{tabular}

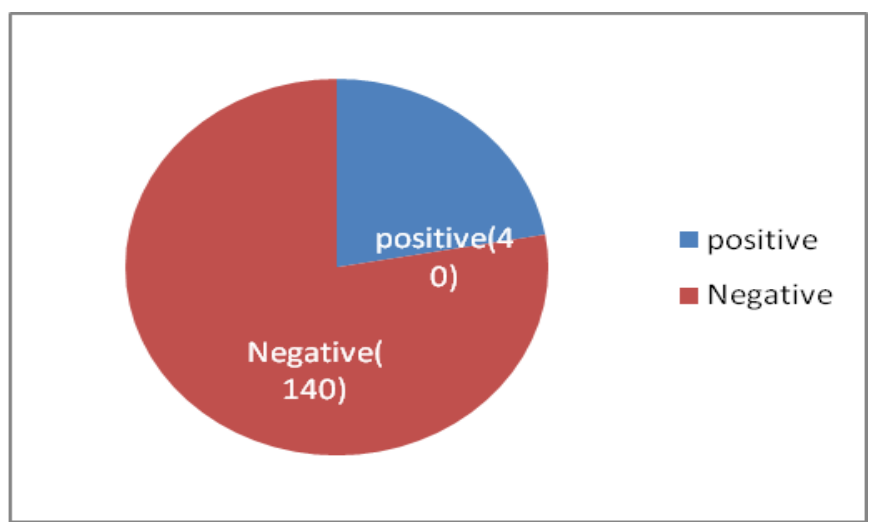


Table.2 Toxoplasma seroprevalence in different age groups $(\mathrm{n}=180)$

\begin{tabular}{|c|c|c|c|c|c|}
\hline Age group & Total & \multicolumn{2}{|c|}{ Positive } & \multicolumn{2}{c|}{ Negative } \\
\hline years & Number & Number & Percentage(\%) & Number & Percentage(\%) \\
\hline upto 20 & 60 & 12 & 20 & 48 & 80 \\
\hline $21-30$ & 60 & 14 & 23.3 & 46 & 76.7 \\
\hline $31-40$ & 44 & 12 & 27.3 & 32 & 72.7 \\
\hline $41-50$ & 12 & 2 & 16.7 & 10 & 83.3 \\
\hline $51-60$ & 4 & - & - & 4 & 100 \\
\hline Total & 180 & 40 & 22.2 & 140 & 77.8 \\
\hline
\end{tabular}

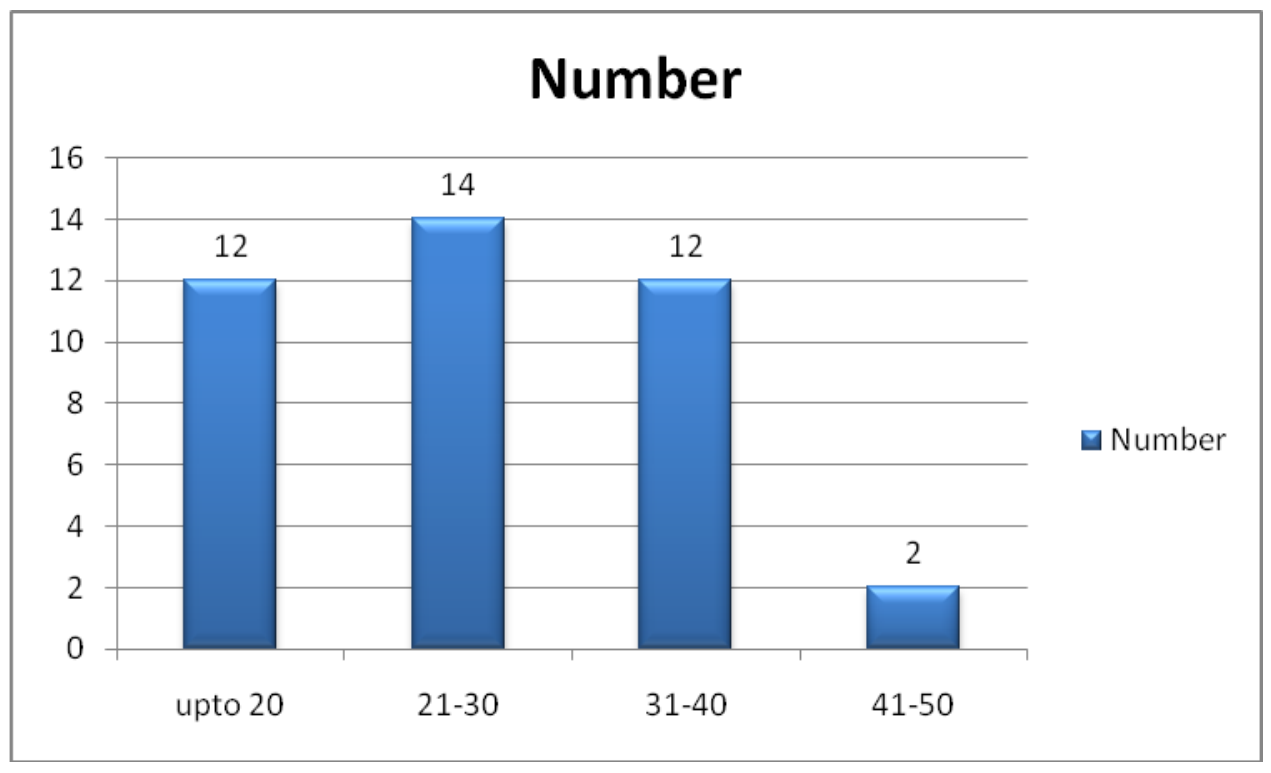

Table.3 Risk factors associated with T.gondiii $\mathrm{IgG}$ positive donors $(\mathrm{n}=40)$

\begin{tabular}{|l|c|c|}
\hline Variables & Total number & Percentage(\%) \\
\hline Age group & 12 & 20 \\
\hline Upto 20 & 14 & 23.3 \\
$21-30$ & 12 & 27.3 \\
$31-40$ & 2 & 16.7 \\
$41-50$ & & \\
\hline Residential place & 30 & 75 \\
Urban & 10 & 25 \\
Rural & & \\
\hline Dietary habits & 10 & 25 \\
Vegetarian & 30 & 75 \\
Non-vegetarian & 12 & 30 \\
\hline Blood group & 10 & 25 \\
A & 6 & 15 \\
B & 12 & 30 \\
AB & & \\
O & & \\
\hline
\end{tabular}


The present study observed none of the female voluntary blood donors reported in the blood bank. A seroprevalence study in antenatal and postnatal clinics can offer better idea of the prevalence in females in the society.

In conclusion, toxoplasma is one of the most important congenitally transmitted infection. Toxoplasma prevalence studies are required throughout the country for the general population and pregnant woman to take preventive measures.

To ensure availability of toxoplasma seronegative blood for transfusion, a national policy of screening of blood and blood products for toxoplasma may need to be included along with other mandatory screening tests.

\section{References}

1. Pinlaor $S$, Ieamviteevanich $K$, Pinlaor $P$, Maleewong W, Pipitgool V. Seroprevalence of specific total immunoglobulin (Ig), IgG and $\operatorname{IgM}$ antibodies to Toxoplasma gondii in blood donors from Loei province, Northeast Thailand. Southeast Asian J Trop Med Public Health 2000; 31: 123 7.

2. Coelho RA, Kobayashi M, Carvalho LB Jr. Prevalence of IgG antibodies specific to Toxoplasma gondii among blood donors in Recife, Northeast Brazil. Rev Inst Med Trop Sao Paulo 2003;45: 229-31.

3. Liu Y, Zheng K, Chen M, Fu L, Du W, Shi Z. Study on detecting antibodies to Toxoplasma gondii in pooled serum of blood donors by Dot-IGSS. Southeast Asian J Trop Med Public Health2001; 32: 558-61.

4. Yang HJ, Jin KN, Park YK, Hong SC, Bae $\mathrm{JM}$, Lee SH, et al. Seroprevalence of toxoplasmosis in the residents of Cheju island, Korea. Korean J Parasitol 2000; 38: 91-3.

5. Zuber P, Jacquier P. Epidemiology of toxoplasmosis: worldwide status. Schweiz Med Wochenschr 1995; 65 (Suppl): 19S-22S.

6. Mittal V, Bhatia R, Sehgal S. Prevalence of Toxoplasma antibodies among women with $\mathrm{BOH}$ and general population in Delhi. J Commun Dis 1990; 22: 223-6.

7. Singh S, Nautiyal BL. Seroprevalence of toxoplasmosis in Kumaon region of India. Indian J Med Res 1991; 93: 2479

8.Elhence P. Agarwal P, Prasad KN, Chaudhary RK (2010) Seroprevalence of $T$. gondii antibodies in North Indian blood donors; implications for transfusion transmissible toxoplasmosis. Transfus Apher Sci 43:37-40.

9. Joshi YR, Vyas S, Joshi KR. Seroprevalence of toxoplasmosis in Jodhpur, India. J Commun Dis 1998; 30: 32-7.

10. Meisheri YV, Mehta S, Patel U. A prospective study of seroprevalence of toxoplasmosis in general population and in HIV/AIDS patients in Bombay, India. J Postgrad Med 1977; 43: 93-7.

11. Sundar, P., A. Mahadevan, R.S. Jayshree, D.K. Subbakrishna and S.K. Shankar. Toxoplasma seroprevalence in healthy voluntary blood donors from urban Karnataka, Bangalore, India. Indian J Med Res 126, July 2007, pp50-55.

12. Singh S, Pandit AJ. Incidence and prevalence of toxoplasmosis in Indian pregnant women: a prospective study. Am J Reprod Immunol 2004; 52: 27683.

13. Yashodhara P, Ramalakshmi BA, Lakshmi V, Krishna TP. Socioeconomic status and prevalence of toxoplasmosis during pregnancy. Indian $J$ Med Microbiol 2004; 22: 241-3.

14. Hall SM. Congenital toxoplasmosis. $\mathrm{Br}$ 
Med J 1992; 305: 291-7.

15.G.Sucilathangam, N.Palaniappan, C.Sreekumar and T.Anna serological survey of toxoplasmosis in a district in TamilNadu J med Res 137.
16. Mohammad Jafari Modrek; Mohammad Mousavi; Ramin Saravani, Toxoplasma gondii Seroprevalence Among Blood Donors in Zahedan, Southeastern Iran, Int J Infect. 2014 July; (1): e21111.

\section{How to cite this article:}

Sundararajan Thangavel, Gomathi Manian and Neelaveni Duraisamy. 2019. Seroprevalence of IgG Antibodies of Toxoplasma in Healthy Voluntary Blood Donors in a Tertiary Care Hospital. Int.J.Curr.Microbiol.App.Sci. 8(03): 576-581. doi: https://doi.org/10.20546/ijcmas.2019.803.070 